\title{
New Results in the Startpoint Theory for Quasipseudometric Spaces
}

\author{
Yaé Ulrich Gaba \\ Department of Mathematics and Applied Mathematics, University of Cape Town, Rondebosch, Cape Town 7701, South Africa \\ Correspondence should be addressed to Yaé Ulrich Gaba; gabayae2@gmail.com
}

Received 22 July 2014; Revised 24 October 2014; Accepted 27 October 2014; Published 17 November 2014

Academic Editor: Aref Jeribi

Copyright (C) 2014 Yaé Ulrich Gaba. This is an open access article distributed under the Creative Commons Attribution License, which permits unrestricted use, distribution, and reproduction in any medium, provided the original work is properly cited.

We give two generalizations of Theorem 35 proved by Gaba (2014). More precisely, we change the structure of the contractive condition; namely, we introduce a function $\Phi$ instead of a simple constant $c$.

\section{Dedicated to Professor Guy A. Degla for his mentorship}

\section{Introduction and Preliminaries}

In [1], we introduced the concept of startpoint and endpoint for set-valued mappings defined on quasipseudometric spaces. As mentioned there, the purpose of this theory is to study fixed point like related properties. In the present, we give more results from the theory. More precisely, we generalize Theorem 35 of [1] by changing the structure of the contractive condition; namely, we introduce a function $\Phi$ instead of a simple constant $c$ (as it appears in the original statement). This new condition is interesting in the sense that it allows us to have a condition involving a functional of the variables and not just the variables themselves. For the convenience of the reader, we will recall some necessary definitions but for a detailed exposé of the definition and examples, the interested reader is referred to [1].

Definition 1. Let $X$ be a nonempty set. A function $d: X \times$ $X \rightarrow[0, \infty)$ is called quasipseudometric on $X$ if

(i) $d(x, x)=0 \forall x \in X$;

(ii) $d(x, z) \leq d(x, y)+d(y, z) \forall x, y, z \in X$.

Moreover, if $d(x, y)=0=d(y, x) \Rightarrow x=y$, then $d$ is said to be a $T_{0}$-quasipseudometric. The latter condition is referred to as the $T_{0}$-condition.
Remark 2. (i) Let $d$ be quasipseudometric on $X$; then the map $d^{-1}$ defined by $d^{-1}(x, y)=d(y, x)$ whenever $x, y \in X$ is also a quasipseudometric on $X$, called the conjugate of $d$. In the literature, $d^{-1}$ is also denoted as $d^{t}$ or $\bar{d}$.

(ii) It is easy to verify that the function $d^{s}$ defined by $d^{s}:=$ $d \vee d^{-1}$, that is, $d^{s}(x, y)=\max \{d(x, y), d(y, x)\}$, defines a metric on $X$ whenever $d$ is a $T_{0}$-quasipseudometric on $X$.

The quasipseudemetric $d$ induces a topology $\tau(d)$ on $X$.

Definition 3. Let $(X, d)$ be a quasipseudometric space. The $d$-convergence of a sequence $\left(x_{n}\right)$ to a point $x$, also called left-convergence and denoted by $x_{n} \stackrel{d}{\rightarrow} x$, is defined in the following way:

$$
x_{n} \stackrel{d}{\longrightarrow} x \Longleftrightarrow d\left(x, x_{n}\right) \longrightarrow 0 .
$$

Similarly, we define the $d^{-1}$-convergence of a sequence $\left(x_{n}\right)$ to a point $x$ or right convergence and denote it by $x_{n} \stackrel{d^{-1}}{\longrightarrow}$ $x$, in the following way:

$$
x_{n} \stackrel{d^{-1}}{\longrightarrow} x \Longleftrightarrow d\left(x_{n}, x\right) \longrightarrow 0 .
$$

Finally, in a quasipseudometric space $(X, d)$, we will say that a sequence $\left(x_{n}\right) d^{s}$-converges to $x$ if it is both left and right 
convergent to $x$, and we denote it as $x_{n} \stackrel{d^{s}}{\rightarrow} x$ or $x_{n} \rightarrow x$ when there is no confusion. Hence

$$
x_{n} \stackrel{d^{s}}{\longrightarrow} x \Longleftrightarrow x_{n} \stackrel{d}{\longrightarrow} x, \quad x_{n} \stackrel{d^{-1}}{\longrightarrow} x .
$$

Definition 4. A sequence $\left(x_{n}\right)$ in quasipseudometric $(X, d)$ is called

(a) left $d$-Cauchy if, for every $\epsilon>0$, there exist $x \in X$ and $n_{0} \in \mathbb{N}$ such that

$$
\forall n \geq n_{0} \quad d\left(x, x_{n}\right)<\epsilon ;
$$

(b) left $K$-Cauchy if, for every $\epsilon>0$, there exists $n_{0} \in \mathbb{N}$ such that

$$
\forall n, k: n_{0} \leq k \leq n \quad d\left(x_{k}, x_{n}\right)<\epsilon
$$

(c) $d^{s}$-Cauchy if, for every $\epsilon>0$, there exists $n_{0} \in \mathbb{N}$ such that

$$
\forall n, k \geq n_{0} \quad d\left(x_{n}, x_{k}\right)<\epsilon .
$$

Dually, we define right $d$-Cauchy and right K-Cauchy sequences.

Definition 5. A quasipseudometric space $(X, d)$ is called

(i) left $K$-complete provided that any left $K$-Cauchy sequence is $d$-convergent,

(ii) left Smyth sequentially complete if any left $K$-Cauchy sequence is $d^{s}$-convergent.

Definition 6. A $T_{0}$-quasipseudometric space $(X, d)$ is called bicomplete provided that the metric $d^{s}$ on $X$ is complete.

As usual, a subset $A$ of a quasipseudometric space $(X, d)$ will be called bounded provided that there exists a positive real constant $M$ such that $d(x, y)<M$ whenever $x, y \in A$.

Let $(X, d)$ be a quasipseudometric space. We set $\mathscr{P}_{0}(X):=$ $2^{X} \backslash\{\emptyset\}$ where $2^{X}$ denotes the power set of $X$. For $x \in X$ and $A, B \in \mathscr{P}_{0}(X)$, we define

$$
\begin{aligned}
& d(x, A)=\inf \{d(x, a), a \in A\}, \\
& d(A, x)=\inf \{d(a, x), a \in A\},
\end{aligned}
$$

and $H(A, B)$ by

$$
H(A, B)=\max \left\{\sup _{a \in A} d(a, B), \sup _{b \in B} d(A, b)\right\} .
$$

Then $H$ is an extended quasipseudometric on $\mathscr{P}_{0}(X)$. Moreover, we know from [2] that the restriction of $H$ to $S_{c l}(X)=\left\{A \subseteq X: A=\left(c l_{\tau(d)} A\right) \cap\left(c l_{\tau\left(d^{-1}\right)} A\right)\right\}$ is an extended $T_{0}$-quasipseudometric. We will denote by $C B(X)$ the collection of all nonempty bounded and $d^{s}$-closed subsets of $X$.

Definition 7 (compare [1]). Let $F: X \rightarrow 2^{X}$ be a set-valued map. An element $x \in X$ is said to be (i) a fixed point of $F$ if $x \in F x$;

(ii) a startpoint of $F$ if $H(\{x\}, F x)=0$;

(iii) an endpoint of $F$ if $H(F x,\{x\})=0$. lemma.

We complete this section by recalling the following

Lemma 8 (compare [1]). Let $(X, d)$ be a quasipseudometric space. For every fixed $x \in X$, the mapping $y \mapsto d(x, y)$ is $\tau(d)$ upper semicontinuous ( $\tau(d)$-usc in short) and $\tau\left(d^{-1}\right)$ lower semicontinuous $\left(\tau\left(d^{-1}\right)\right.$-lsc in short). For every fixed $y \in X$, the mapping $x \mapsto d(x, y)$ is $\tau(d)$-lsc and $\tau\left(d^{-1}\right)$-usc.

\section{Main Results}

We commence this section with the main result of this paper.

Theorem 9. Let $(X, d)$ be a left $K$-complete quasipseudometric space. Let $T: X \rightarrow C B(X)$ be a set-valued map and define $f: X \rightarrow \mathbb{R}$ as $f(x)=H(\{x\}$, Tx $)$. Let $\Phi:[0, \infty) \rightarrow[0,1)$ be a function such that $\limsup _{r \rightarrow t^{+}} \Phi(r)<1$ for each $t \in[0, \infty)$. Moreover, assume that for any $x \in X$ there exists $y \in T x$ satisfying

$$
H(\{y\}, T y) \leq \Phi(H(\{x\},\{y\})) H(\{x\},\{y\}),
$$

and then $T$ has a startpoint.

Proof. First observe that, since $\Phi(H(\{x\},\{y\}))<1$ for any $x, y \in X$, it follows that $2-\Phi(H(\{x\},\{y\}))>1$ for any $x, y \in$ $X$, and hence

$$
H(\{x\},\{y\}) \leq[2-\Phi(H(\{x\},\{y\}))] H(\{x\}, T x),
$$

for any $x \in X$ and $y \in T x$.

For any initial $x_{0} \in X$, there exists (for all actually) $x_{1} \in$ $T x_{0} \subseteq X$ such that

$$
H\left(\left\{x_{0}\right\},\left\{x_{1}\right\}\right) \leq\left[2-\Phi\left(H\left(\left\{x_{0}\right\},\left\{x_{1}\right\}\right)\right)\right] H\left(\left\{x_{0}\right\}, T x_{0}\right) .
$$

From (9) we get

$$
\begin{aligned}
H\left(\left\{x_{1}\right\}, T x_{1}\right) \leq & \Phi\left(H\left(\left\{x_{0}\right\},\left\{x_{1}\right\}\right)\right) H\left(\left\{x_{0}\right\},\left\{x_{1}\right\}\right) \\
\leq & \Phi\left(H\left(\left\{x_{0}\right\},\left\{x_{1}\right\}\right)\right) \\
& \times\left[2-\Phi\left(H\left(\left\{x_{0}\right\},\left\{x_{1}\right\}\right)\right)\right] H\left(\left\{x_{0}\right\}, T x_{0}\right) \\
= & \Psi\left(H\left(\left\{x_{0}\right\},\left\{x_{1}\right\}\right)\right) H\left(\left\{x_{0}\right\}, T x_{0}\right),
\end{aligned}
$$

where $\Psi:[0, \infty) \rightarrow[0,1)$ is defined by

$$
\Psi(t)=\Phi(t)(2-\Phi(t))
$$

Now choosing $x_{2} \in F x_{1} \subseteq X$, we have that

$$
H\left(\left\{x_{1}\right\},\left\{x_{2}\right\}\right) \leq\left[2-\Phi\left(H\left(\left\{x_{1}\right\},\left\{x_{2}\right\}\right)\right)\right] H\left(\left\{x_{1}\right\}, T x_{1}\right),
$$


and from (9) we get

$$
H\left(\left\{x_{2}\right\}, T x_{2}\right) \leq \Psi\left(H\left(\left\{x_{1}\right\},\left\{x_{2}\right\}\right)\right) H\left(\left\{x_{1}\right\}, T x_{1}\right) .
$$

Continuing this process, we obtain a sequence $\left(x_{n}\right)$ where $x_{n+1} \in T x_{n} \subseteq X$, with

$$
\begin{aligned}
& H\left(\left\{x_{n}\right\},\left\{x_{n+1}\right\}\right) \\
& \quad \leq\left[2-\Phi\left(H\left(\left\{x_{n}\right\},\left\{x_{n+1}\right\}\right)\right)\right] H\left(\left\{x_{n}\right\}, T x_{n}\right) \\
& H\left(\left\{x_{n+1}\right\}, T x_{n+1}\right) \leq \Psi\left(H\left(\left\{x_{n}\right\},\left\{x_{n+1}\right\}\right)\right) H\left(\left\{x_{n}\right\}, T x_{n}\right) \\
& \quad n=1,2, \ldots
\end{aligned}
$$

For simplicity, denote $d_{n}:=H\left(\left\{x_{n}\right\},\left\{x_{n+1}\right\}\right)$ and $D_{n}:=$ $H\left(\left\{x_{n}\right\}, T x_{n}\right)$ for all $n \geq 0$. So from (17) we can write

$$
D_{n+1} \leq \Psi\left(d_{n}\right) D_{n} \leq D_{n}
$$

for all $n \geq 0$. Hence $\left(D_{n}\right)$ is a strictly decreasing sequence and hence there exists $\delta \geq 0$ such that

$$
\lim _{n \rightarrow \infty} D_{n}=\delta
$$

From (16), it is easy to see that

$$
d_{n}<2 D_{n}
$$

Thus the sequence $\left(d_{n}\right)$ is bounded and so there is $d \geq 0$ such that $\limsup _{n \rightarrow \infty} d_{n}=d$ and hence a subsequence $\left(d_{n_{k}}\right)$ of $\left(d_{n}\right)$ such that $\lim _{k \rightarrow \infty} d_{n_{k}}=d^{+}$. From (17) we have $D_{n_{k}+1} \leq$ $\Psi\left(d_{n_{k}}\right) D_{n_{k}}$ and thus

$$
\begin{aligned}
\delta & =\limsup _{k \rightarrow \infty} D_{n_{k}+1} \\
& \leq\left(\limsup _{k \rightarrow \infty} \Psi\left(d_{n_{k}}\right)\right)\left(\limsup _{k \rightarrow \infty} D_{n_{k}}\right) \\
& \leq \limsup _{d_{n_{k}} \rightarrow d^{+}} \Psi\left(d_{n_{k}}\right) \delta .
\end{aligned}
$$

This together with the fact $\limsup _{r \rightarrow t^{+}} \Phi(r)<1$ for each $t \in[0, \infty)$ implies that $\delta=0$. Then from (19) and (20) we derive that $\lim _{n \rightarrow \infty} d_{n}=0$.

Claim 1. $\left(x_{n}\right)$ is a left $K$-Cauchy sequence.

Now let $\alpha:=\lim \sup _{d_{n} \rightarrow 0^{+}} \Psi\left(d_{n}\right)$ and $q$ such that $\alpha<q<$ 1 . This choice of $q$ is always possible since $\alpha<1$. Then there is $n_{0}$ such that $\Psi\left(d_{n}\right)<q$ for all $n \geq n_{0}$. So from (18) we have $D_{n+1} \leq q D_{n}$ for all $n \geq n_{0}$. Then by induction we get $D_{n} \leq$ $q^{n-n_{0}} D_{n_{0}}$ for all $n \geq n_{0}+1$. Combining this and inequality (20) we get

$$
\sum_{k=n_{0}}^{m} d\left(x_{k}, x_{k+1}\right) \leq 2 \sum_{k=n_{0}}^{m} q^{k-n_{0}} D_{n_{0}} \leq 2 \frac{1}{1-q} D_{n_{0}}
$$

for all $m>n \geq n_{0}+1$. Hence $\left(x_{n}\right)$ is a left $K$-Cauchy sequence.
According to the left $K$-completeness of $(X, d)$, there exists $x^{*} \in X$ such that $x_{n} \stackrel{d}{\rightarrow} x^{*}$.

Claim 2. $x^{*}$ is a startpoint of $T$.

Observe that the sequence $D_{n}=\left(f x_{n}\right)=\left(H\left(\left\{x_{n}\right\}, T x_{n}\right)\right)$ converges to 0 . Since $f$ is $\tau(d)$-lower semicontinuous (as supremum of $\tau(d)$-lower semicontinuous functions), we have

$$
0 \leq f\left(x^{*}\right) \leq \liminf _{n \rightarrow \infty} f\left(x_{n}\right)=0 .
$$

Hence $f\left(x^{*}\right)=0$; that is, $H\left(\left\{x^{*}\right\}, T x^{*}\right)=0$.

This completes the proof.

We give below an example to illustrate the theorem.

Example 10. Let $X=[0,1]$ and $d: X \times X \rightarrow \mathbb{R}$ be the mapping defined by $d(a, b)=\max \{a-b, 0\}$. Then $d$ is a $T_{0}$-quasipseudometric on $X$. Observe that any left $K$-Cauchy sequence in $(X, d)$ is $d$-convergent to 0 . Indeed, if $\left(x_{n}\right)$ is a left $K$-Cauchy sequence, for every $\epsilon>0$, there exists $n_{0} \in \mathbb{N}$ such that

$$
\forall n, k: n_{0} \leq k \leq n \quad d\left(x_{k}, x_{n}\right)<\epsilon .
$$

This entails that $\forall n: n_{0}<n$

$$
d\left(0, x_{n}\right) \leq d\left(0, x_{n-1}\right)+d\left(x_{n-1}, x_{n}\right)=0+d\left(x_{n-1}, x_{n}\right)<\epsilon .
$$

Hence $d\left(0, x_{n}\right) \rightarrow 0$; that is, $x_{n} \stackrel{d}{\rightarrow} 0$. Therefore $(X, d)$ is left $K$-complete. Let $T: X \rightarrow C B(X)$ be such that

$$
T(x)= \begin{cases}\left\{\frac{1}{2} x^{2}\right\}, & \text { for } x \in\left[0, \frac{15}{32}\right) \cup\left(\frac{15}{32}, 1\right], \\ \left\{\frac{17}{96}, \frac{1}{4}\right\}, & \text { for } x=\frac{15}{32} .\end{cases}
$$

Let $\Phi:[0, \infty) \rightarrow[0,3 / 4) \subset[0,1)$ be defined by

$$
\Phi(r)= \begin{cases}\frac{3}{2} r, & \text { for } r \in\left[0, \frac{7}{24}\right) \cup\left(\frac{7}{24}, \frac{1}{2}\right) \\ \frac{425}{768}, & \text { for } r=\frac{7}{24} \\ \frac{1}{2}, & \text { for } r \in\left[\frac{1}{2}, \infty\right) .\end{cases}
$$

An explicit computation of $f(x)=H(\{x\}, T x)$ gives

$$
f(x)= \begin{cases}x-\frac{1}{2} x^{2}, & \text { for } x \in\left[0, \frac{15}{32}\right) \cup\left(\frac{15}{32}, 1\right], \\ \frac{28}{96}, & \text { for } x=\frac{15}{32} .\end{cases}
$$

Moreover, for each $x \in[0,15 / 32) \cup(15 / 32,1], y=$ $(1 / 2) x^{2}$ and we have

$$
H(\{y\}, T y) \leq \Phi(H(\{x\},\{y\})) H(\{x\},\{y\}) .
$$

Of course inequality (29) also holds in the case of $x=15 / 32$ and $y=17 / 96$. Therefore, all assumptions of Theorem 9 are satisfied and the endpoint of $T$ is $x=0$. 
Remark 11. In fact, every sequence in $(X, d) d$-converges to 0 .

Corollary 12. Let $(X, d)$ be a right $K$-complete quasipseudometric space. Let $T: X \rightarrow C B(X)$ be a set-valued map and define $f: X \rightarrow \mathbb{R}$ as $f(x)=H(T x,\{x\})$. Let $\Phi:[0, \infty) \rightarrow$ $[0,1)$ be a function such that $\lim _{\sup _{r \rightarrow t^{+}}} \Phi(r)<1$ for each $t \in[0, \infty)$. Moreover, assume that for any $x \in X$ there exists $y \in$ Tx satisfying

$$
H(T y,\{y\}) \leq \Phi(H(\{y\},\{x\})) H(\{y\},\{x\}),
$$

and then $T$ has an endpoint.

Corollary 13. Let $(X, d)$ be a bicomplete quasipseudometric space. Let $T: X \rightarrow C B(X)$ be a set-valued map and $f: X \rightarrow \mathbb{R}$ defined by $f(x)=H^{s}(T x,\{x\})=$ $\max \{H(T x,\{x\}), H(\{x\}, T x)\}$. If there exists $c \in(0,1)$ such that for all $x \in X$ there exists $y \in F x$ satisfying

$$
H^{s}(\{y\}, F y) \leq \min \{\Phi(a) a, \Phi(b) b\},
$$

where $a=H(\{y\},\{x\})$ and $b=H(\{x\},\{y\})$, then $T$ has a fixed point.

Proof. We give here the main idea of the proof. Observe that inequality (31) guarantees that the sequence $\left(x_{n}\right)$ constructed in the proof of Theorem 9 is a $d^{s}$-Cauchy sequence and hence $d^{s}$-converges to some $x^{*}$. Using the fact that $f$ is $\tau\left(d^{s}\right)$ lower semicontinuous (as supremum of $\tau\left(d^{s}\right)$-continuous functions), we have

$$
0 \leq f\left(x^{*}\right) \leq \liminf _{n \rightarrow \infty} f\left(x_{n}\right)=0 .
$$

Hence $f\left(x^{*}\right)=0$; that is, $H\left(\left\{x^{*}\right\}, T x^{*}\right)=0=$ $H\left(T x^{*},\left\{x^{*}\right\}\right)$, and we are done.

The following theorem is the second generalization that we propose.

Theorem 14. Let $(X, d)$ be a left $K$-complete quasipseudometric space. Let $T: X \rightarrow C B(X)$ be a set-valued map and define $f: X \rightarrow \mathbb{R}$ as $f(x)=H(\{x\}, T x)$. Let $b:[0, \infty) \rightarrow$ $[a, 1), a>0$ be a nondecreasing function. Let $\Phi:[0, \infty) \rightarrow$ $[0,1)$ be a function such that $\Phi(t)<b(t)$ for each $t \in[0, \infty)$ and $\lim \sup _{r \rightarrow t^{+}} \Phi(r)<\limsup _{r \rightarrow t^{+}} b(r)$ for each $t \in[0, \infty)$. Moreover, assume that for any $x \in X$ there exists $y \in T x$ satisfying

$$
H(\{y\}, T y) \leq \Phi(H(\{x\},\{y\})) H(\{x\},\{y\}),
$$

and then $T$ has a startpoint.

Proof. Observe that because $b(H(\{x\},\{y\}))<1$ for all $x, y \in$ $X$,

$$
b(H(\{x\},\{y\})) H(\{x\},\{y\}) \leq H(\{x\}, T x),
$$

for any $y \in T x$. Let $x_{0} \in X$ be arbitrary. Then we can choose $x_{1} \in T x_{0}$ such that

$$
\begin{aligned}
& b\left(H\left(\left\{x_{0}\right\},\left\{x_{1}\right\}\right)\right) H\left(\left\{x_{0}\right\},\left\{x_{1}\right\}\right) \leq H\left(\left\{x_{0}\right\}, T x_{0}\right) \\
& H\left(\left\{x_{1}\right\}, T x_{1}\right) \leq \Phi\left(H\left(\left\{x_{0}\right\},\left\{x_{1}\right\}\right)\right) H\left(\left\{x_{0}\right\},\left\{x_{1}\right\}\right) .
\end{aligned}
$$

Define the function $\Psi:[0, \infty) \rightarrow[0,1)$ by $\Psi(t)=$ $\Phi(t) / b(t)$. Hence (35) and (36) together sum to

$$
H\left(\left\{x_{1}\right\}, T x_{1}\right) \leq \Psi\left(H\left(\left\{x_{0}\right\},\left\{x_{1}\right\}\right)\right) H\left(\left\{x_{0}\right\}, T x_{0}\right) .
$$

Now we choose $x_{2} \in T x_{1}$ such that

$$
\begin{aligned}
& b\left(H\left(\left\{x_{1}\right\},\left\{x_{2}\right\}\right)\right) H\left(\left\{x_{1}\right\},\left\{x_{2}\right\}\right) \leq H\left(\left\{x_{1}\right\}, T x_{1}\right), \\
& H\left(\left\{x_{2}\right\}, T x_{2}\right) \leq \Phi\left(H\left(\left\{x_{1}\right\},\left\{x_{2}\right\}\right)\right) H\left(\left\{x_{1}\right\},\left\{x_{2}\right\}\right),
\end{aligned}
$$

which lead to

$$
H\left(\left\{x_{2}\right\}, T x_{2}\right) \leq \Psi\left(H\left(\left\{x_{1}\right\},\left\{x_{2}\right\}\right)\right) H\left(\left\{x_{1}\right\}, T x_{1}\right) .
$$

Continuing this process, we get an iterative sequence $\left(x_{n}\right)$ where $x_{n+1} \in T x_{n} \subseteq X$ and, denoting $d_{n}=H\left(\left\{x_{n}\right\},\left\{x_{n+1}\right\}\right)$ and $D_{n}=H\left(\left\{x_{n}\right\}, T x_{n}\right)$ for all $n \geq 0$, we can write that

$$
b\left(d_{n}\right) d_{n} \leq D_{n}, \quad D_{n+1} \leq \Phi\left(d_{n}\right) d_{n}
$$

for all $n \geq 0$. Hence

$$
D_{n+1} \leq \Psi\left(d_{n}\right) D_{n}
$$

If $D_{k}=0$ for some $k \geq 0$, then we trivially have $\lim _{n \rightarrow \infty} D_{n}=0$ and the conclusion is immediate. So without loss of generality, we can assume that $D_{n}>0$ for all $n \geq 0$ and, from (41), we have $D_{n+1}<D_{n}$ for all $n \geq 0$.

Observe also that if, for some $n, d_{n} \leq d_{n+1}$, we are led to a contradiction. Indeed from (40) and using the fact that the function $b$ is nondecreasing, we have

$$
d_{n} \leq d_{n+1} \leq \frac{\Phi\left(d_{n}\right)}{b\left(d_{n+1}\right)} d_{n} \leq \frac{\Phi\left(d_{n}\right)}{b\left(d_{n}\right)} d_{n}=\Psi\left(d_{n}\right) d_{n}<d_{n} .
$$

Hence $d_{n+1}<d_{n}$ for all $n \geq 0$. Thus there exist $\delta \geq 0$ and $d \geq 0$ such that $\lim _{n \rightarrow \infty} D_{n}=\delta$ and $\lim _{n \rightarrow \infty} d_{n}=d$. From (41), we get

$$
\delta \leq\left(\limsup _{n \rightarrow \infty} \Psi\left(d_{n}\right)\right) \delta=\limsup _{d_{n} \rightarrow d^{+}} \Psi\left(d_{n}\right) \delta,
$$

and hence $\delta=0$ (since $\limsup _{r \rightarrow t^{+}} \Phi(r)<1$ for all $t \epsilon$ $[0, \infty))$. Moreover, since

$$
a d_{n} \leq b\left(d_{n}\right) d_{n} \leq D_{n} \leq \Psi\left(d_{n-1}\right) D_{n-1},
$$

this forces $d$ to be 0 ; that is, $\lim _{n \rightarrow \infty} d_{n}=0$.

Furthermore, setting $\alpha:=\lim \sup _{d_{n} \rightarrow 0^{+}} \Psi\left(d_{n}\right)$ and letting $q>0$ be a positive number such that $\alpha<q<1$, there is $n_{0}$ such that $\Psi\left(d_{n}\right)<q$ for all $n \geq n_{0}$. Hence from (41) and (44), we get

$$
a d_{n} \leq \Psi\left(d_{n-1}\right) D_{n-1} \leq q D_{n-1} \leq \cdots \leq q^{n-n_{0}} D_{n_{0}},
$$

for all $n \geq n_{0}$. So $d_{n} \leq(1 / a) q^{n-n_{0}} D_{n_{0}}$ for all $n \geq n_{0}$. Using a similar argument as the one used in the proof of Theorem 9, we conclude that $\left(x_{n}\right)$ is a left $K$-Cauchy sequence and that its limit point is a starpoint of $T$. 
Example 15. Let $X=[0,1)$ and $d: X \times X \rightarrow \mathbb{R}$ be the mapping defined by $d(a, b)=\max \{a-b, 0\}$. Then $d$ is a $T_{0}$-quasipseudometric on $X$. We know that $(X, d)$ is left $K$ complete. Let $T: X \rightarrow C B(X)$ be such that

$$
T(x)=\left\{\frac{1}{2} x^{2}\right\} .
$$

Let $\Phi:[0, \infty) \rightarrow[0,3 / 4) \subset[0,1)$ be defined by

$$
\Phi(r)= \begin{cases}\frac{3}{2} r, & \text { for } r \in\left[0, \frac{1}{2}\right) \\ 0, & \text { for } r \in\left[\frac{1}{2}, \infty\right) .\end{cases}
$$

Let $b:[0, \infty) \rightarrow[2 / 3,1)$ be defined by

$$
b(t)= \begin{cases}\frac{5}{6} t, & \text { for } t \in\left[0, \frac{1}{2}\right) \\ \frac{1}{2}, & \text { for } t \in\left[\frac{1}{2}, \infty\right) .\end{cases}
$$

An explicit computation of $f(x)=H(\{x\}, T x)$ gives

$$
f(x)=x-\frac{1}{2} x^{2}, \quad \text { for } x \in[0,1) .
$$

Moreover, for each $x \in[0,1)$, there exists $y=(1 / 2) x^{2}$ and we have

$$
\begin{aligned}
H(\{y\}, T y) & \leq \frac{3}{2} H(\{x\},\{y\}) H(\{x\},\{y\}) \\
& =\Phi(H(\{x\},\{y\})) H(\{x\},\{y\}) .
\end{aligned}
$$

Therefore, all assumptions of Theorem 14 are satisfied and the endpoint of $T$ is $x=0$.

Corollary 16. Let $(X, d)$ be a right $K$-complete quasipseudometric space. Let $T: X \rightarrow C B(X)$ be a set-valued map and define $f: X \rightarrow \mathbb{R}$ as $f(x)=H^{-1}(\{x\}, T x)$. Let $b:[0, \infty) \rightarrow[a, 1), a>0$ be a nondecreasing function. Let $\Phi:[0, \infty) \rightarrow[0,1)$ be a function such that $\Phi(t)<b(t)$ for each $t \in[0, \infty)$ and $\lim \sup _{r \rightarrow t^{+}} \Phi(r)<\limsup _{r \rightarrow t^{+}} b(r)$ for each $t \in[0, \infty)$. Moreover, assume that for any $x \in X$ there exists $y \in T x$ satisfying

$$
H^{-1}(\{y\}, T y) \leq \Phi\left(H^{-1}(\{x\},\{y\})\right) H^{-1}(\{x\},\{y\}),
$$

$\Phi(t)<b(t)$, and $\lim \sup _{r \rightarrow t^{+}} \Phi(r)<\limsup _{r \rightarrow t^{+}} b(r)$ for each $t \in[0, \infty)$. Moreover, assume that for any $x \in X$ there exists $y \in T x$ satisfying and then $T$ has an endpoint.

Corollary 17. Let $(X, d)$ be a bicomplete quasipseudometric space. Let $T: X \rightarrow C B(X)$ be a set-valued map and define $f: X \rightarrow \mathbb{R}$ as $f(x)=H^{s}(\{x\}, T x)$. Let $b:[0, \infty) \rightarrow[a, 1)$, $a>0$ be a nondecreasing function. Let $\Phi:[0, \infty) \rightarrow[0,1)$ be a function such that $\Phi(t)<b(t)$ and $\limsup _{r \rightarrow t^{+}} \Phi(r)<$ $\limsup _{r \rightarrow t^{+}} b(r)$ for each $t \in[0, \infty)$. Moreover, assume that for any $x \in X$ there exists $y \in T x$ satisfying

$$
H^{s}(\{y\}, T y) \leq \min \{\Phi(a) a, \Phi(b) b\},
$$

where $a=H(\{x\},\{y\})$ and $b=H^{-1}(\{x\},\{y\})$. Then $T$ has $a$ fixed point.
Remark 18. All the results given remain true when we replace accordingly the bicomplete quasipseudometric space $(X, d)$ by a left Smyth sequentially complete/left $K$-complete or a right Smyth sequentially complete/right $K$-complete space.

\section{Conflict of Interests}

The author declares that there is no conflict of interests regarding the publication of this paper.

\section{References}

[1] Y. U. Gaba, "Startpoints and $(\alpha, \gamma)$-contractions in quasipseudometric spaces," Journal of Mathematics, vol. 2014, Article ID 709253, 8 pages, 2014.

[2] H.-P. Künzi and C. Ryser, "The Bourbaki quasi-uniformity," Topology Proceedings, vol. 20, pp. 161-183, 1995. 


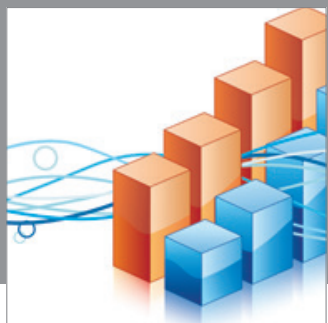

Advances in

Operations Research

mansans

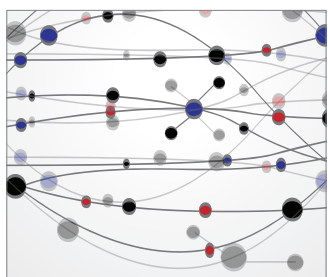

The Scientific World Journal
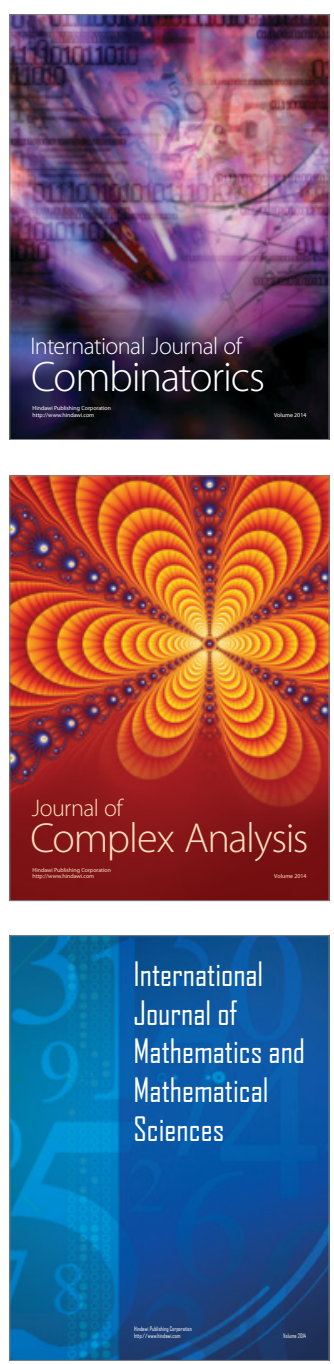
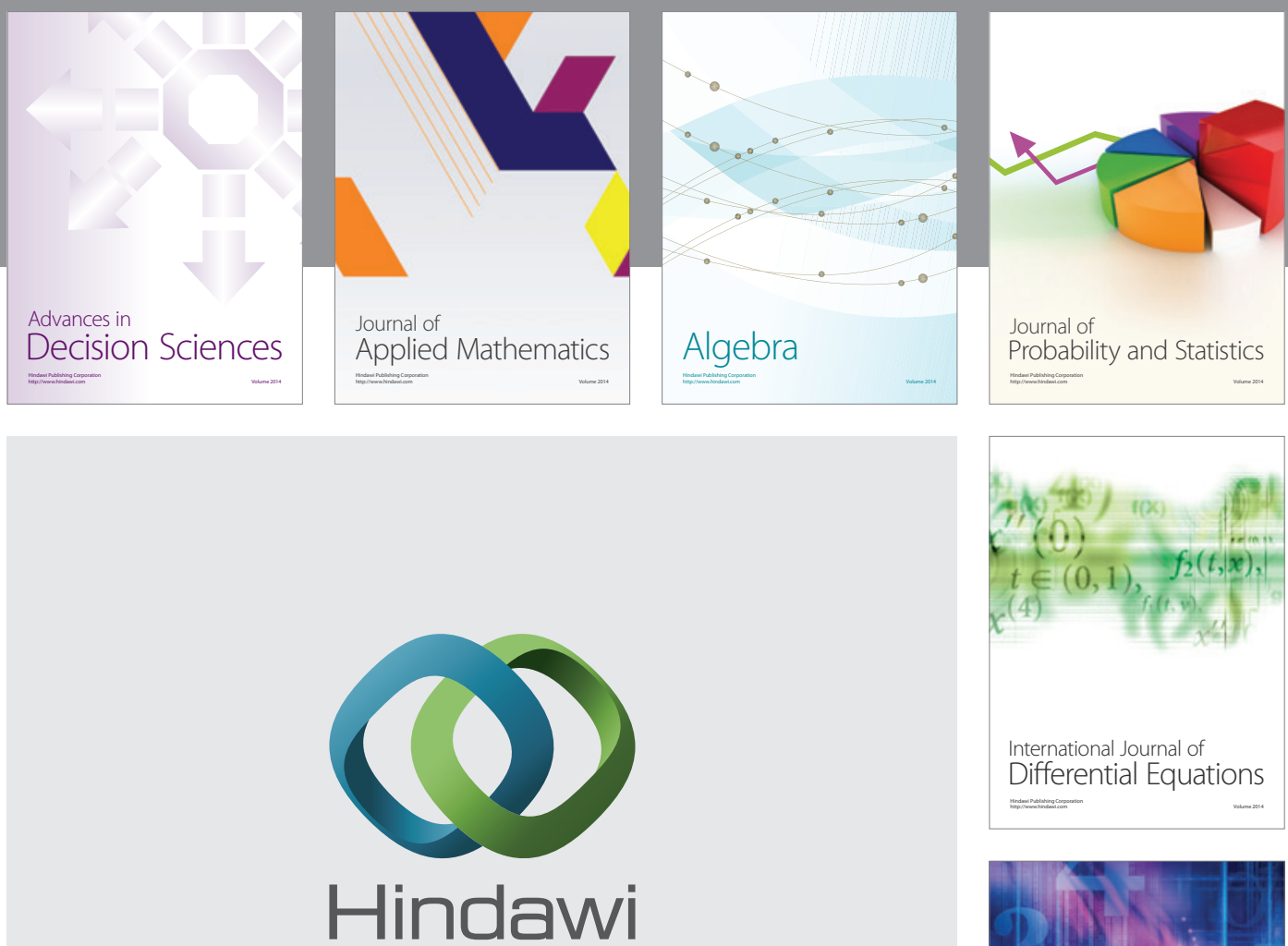

Submit your manuscripts at http://www.hindawi.com
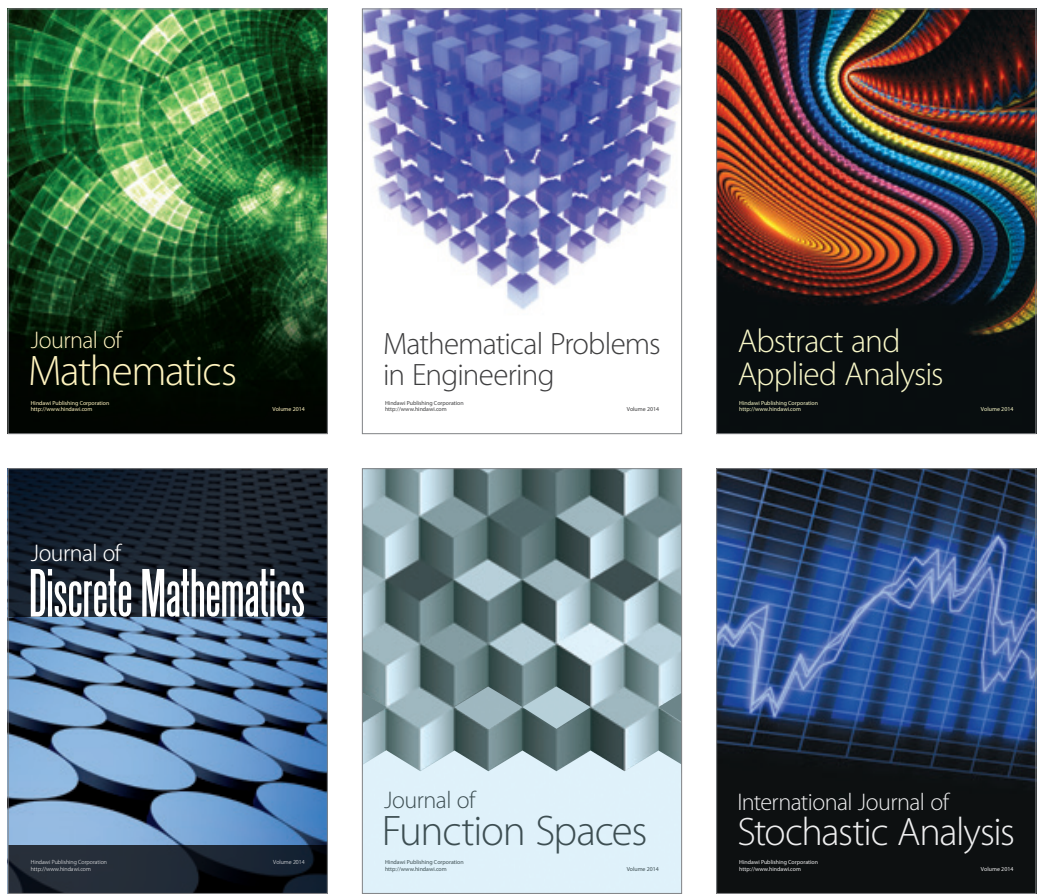

Journal of

Function Spaces

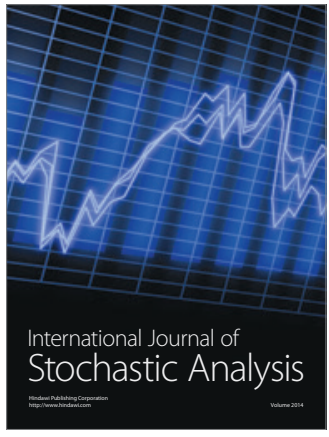

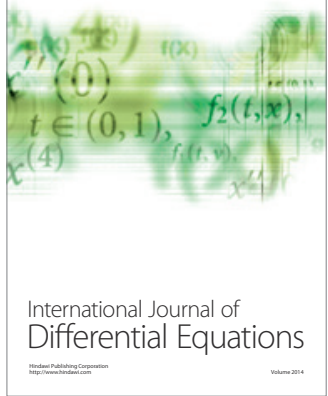
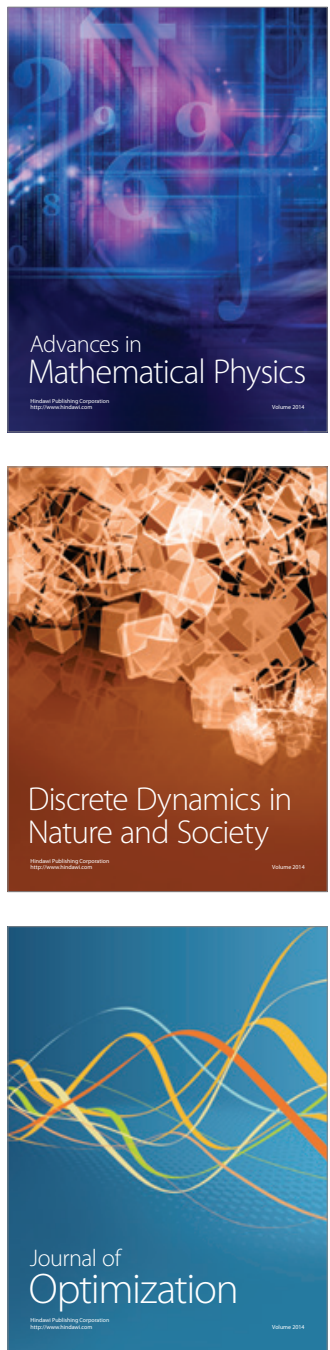